Meta

Journal des traducteurs

Translators' Journal

\title{
Le vocabulaire de la buanderie
}

\section{Erich Oehnel}

Volume 14, numéro 4, décembre 1969

URI : https://id.erudit.org/iderudit/003754ar

DOI : https://doi.org/10.7202/003754ar

Aller au sommaire du numéro

Éditeur(s)

Les Presses de l'Université de Montréal

ISSN

0026-0452 (imprimé)

1492-1421 (numérique)

Découvrir la revue

Citer cet article

Oehnel, E. (1969). Le vocabulaire de la buanderie. Meta, 14(4), 211-218.

https://doi.org/10.7202/003754ar d'utilisation que vous pouvez consulter en ligne.

https://apropos.erudit.org/fr/usagers/politique-dutilisation/ 


\section{LE VOCABULAIRE DE LA BUANDERIE}

Personnel

Effectifs (l'effectif)

Direction

Directeur de la buanderie et de la lingerie

Directeur adjoint de la buanderie et de la lingerie

Chef de la buanderie

Sous-chef de la buanderie

\section{Cadres}

Chef de service

Contremaître

\section{Équipe}

Chef d'équipe

Maître blanchisseur

Blanchisseur

Aide-blanchisseur

Conducteur de séchoir

Conducteur d'essoreuse

Chef-buandier

Presseur/presseuse (de linge en forme)

Marqueuse

Calandreur/calandreuse

Buandier/buandière

Aide-buandier/aide-buandière

Maîtresse lingère

Tailleuse
Staff

Total work force

\section{Management}

Director of laundry and linen supply

Assistant director of laundry and linen supply

Laundry manager

Assistant laundry manager

Supervisory staff

Department supervisor

Foreman

\section{Labor}

Group leader

Head washman

Washman

Washfloor helper

Tumbler man

Extractor man

Floor foreman

Laundry press operator

Marking machine operator

Flatwork ironer operator

Laundry worker (male, female)

Laundry helper (male, female)

Sewing room supervisor

Tailoress 
Couturière

Lingère

Aide-lingère

Terminologie générale

Buanderie

Blanchisserie

Blanchisserie

Buanderie d'établissement

Buanderie d'hôpital

Blanchissage commercial

Blanchissage d'établissement

Blanchissage de famille

Implantation des machines

Disposition des lieux

Salle de lavage

Aire de pré-repassage

Salle de stockage du linge

Organigramme

Service (ou section) des achats

Service (ou section) de buanderie

Service (ou section) de raccommodage

Service (ou section) de remplacement

Service (ou section) de distribution

Service (ou section) d'entretien

Organisation du travail

Achats

Calandrage

Circuit

Tri

Contrôle du linge

Couture à la main

Couture à la machine

Distribution

Empaquetage

Empilage

Essangeage

Lavage

Marquage

Pliage

Pressage

Ramassage

Raccommodage

Remplacement

Repassage

Séchage

Stockage du linge

Calandre

Regarnir la calandre

Réglage de vitesse
Seamstress

Linen room supervisor

Linen maid

Basic terminology

Laundry (institutional type)

Laundry (commercial type)

Commercial laundry (v. laundry)

Institutional laundry

Hospital laundry

Commercial laundry work

Institutional laundry work

Family work

Plant lay-out (machinery)

Plant lay-out (buildings)

Washfloor

Folding and shaking area

Linen storage room

Organizational chart

Purchasing department

Laundry department

Repair department

Replacement department

Distribution department

Maintenance department

Production scheduling

Purchasing

Pressing operation (flatwork ironer)

Circulation

Classification

Linen control

Hand sewing

Machine sewing

Linen distribution

Packaging

Stacking

Prespotting

Washing operation

Marking operation

Folding

Pressing operation (press)

Collecting

Linen repair

Replacement

Ironing operation (by hand)

Drying operation

Linen storage

Flatwork ironer

Change of flatwork ironer roll-padding and roll-over

Speed control 
Relevage des rouleaux

Ruban de calandre

Bandes d'approvisionnement

Bandes de retour

Coton d'amiante

Bourrure

Bourrure de laine d'acier

Molleton

Recouvrement de coton

Cire pour calandre

Tablier

Toit d'évacuation

Déployeuse

Plieuse automatique

Plieuse transversale

Empileuse

Plieuse-empileuse

Empileuse automatique

Avec agrafes

Essoreuse

Couvercle

Centrifugeuse-essoreuse

Presse essoreuse à rouleaux

Essoreuse «sans courbe »

Frein automatique

Compte-minutes

Panier

Période d'essorage

Essoreuse à courroie

Essoreuse autonome

Tambour à essoreuse

Régler la minuterie

Fer à repasser

Fer à vapeur

Fer électrique

Lessiveuse

Lessiveuse-essoreuse

Lessiveuse à deux compartiments

Lessiveuse à déchargement automatique

Lessiveuse à alimentation automatique

Lessiveuse à chargement frontal

Lessiveuse à chargement latéral

Lessiveuse en bois

Lessiveuse en acier inoxydable

Lessiveuse en monel

A l'épreuve de la corrosion

Cylindre de lessiveuse

Arêtes

Indicateur de niveau d'eau

Cuve extérieure

Commande à deux vitesses

Machine à marquer

Mannequin de séchage
Release of roll-pressure

Flatwork ironer tape

Feed ribbon

Return ribbon

Asbestos cloth

Padding

Steelwool padding

Knitted padding

Covercloth, cotton

Flatwork ironer wax

Flatwork ironer apron

Canopy

Spreader

Automatic folder

Cross folder

Stacker

Combined folder and stacker

Automatic stacker

Clipper laced

Extractor

Lid

Centrifugal extractor

Pressure-type hydraulic extractor

Open top extractor

Automatic brakes

Time setter

Extractor basket

Extraction time

Belt driven extractor

Motor driven extractor

Extractor basket

Set-time on dial

Hand iron

Steam iron

Electrical iron

Washer

Washer-extractor

Two pocket washer

Automatic unloading washer

Washer with automatic feeder

Front end loading washer

Side loading washer

Wooden washer

Stainless steel washer

Monel steel washer

Corrosion-proof

Washwheel

Ribs

Water level indicator

Outer shell

Two speed choice

Marking machine

Bodyform, body finishing unit 
Planche à détacher

Planche à repasser

Presse

Presse universelle

Presse tout usage

Presse pour blouses de travail

Presse à vêtements

Ensemble de presses de finition

Compresseur

Boule à vapeur

Coque à vapeur

Séchoir

Séchoir-démêloir

Séchoir de conditionnement

Séchoir de séchage complet

Tunnel de conditionnement

Alimenté par bande transporteuse

Chauffage au gaz

Séchoir de conditionnement et de séchage complet

Minuterie

Lampe témoin

Tambour intérieur

Appareillage et accessoires

Balance à plateau

Baril, barillet

Décrasseuse

Brosse à détacher

Chariot de lavage

Chariot de linge

Chariot en toile

Cuvier sur roue

Chariot tout usage

Cuvier

Cintre

Cuiseur d'empois

Epingle à filet

Filet

Jarre de grès

Récipient

Seau

Table d'empilage

Trousse pour détacher

Vide

Fiche

Fonctionnement de la lessiveuse

Charge

Chargement

Charge insuffisante

Charge excessive
Spotting board

Ironing board

Laundry press

All-purpose press

Utility press

Uniform press

Wearing apparel press

Finishing unit

Aircompressor

Puff iron (ball type)

Puff iron (spheric type)

Dryer

Shake-out tumbler

Conditioning tumbler

Drying tumbler

Continuous flow conditioning tumbler

Conveyor fed tumbler

Gas heated

Combined drying and conditioning tumbler

Timer

Signal lamp

Drum

Additional equipment

Platform scale

Drum, small drum

Recipient for kier-boils

Heatable receptacle for kier-boils

Spotting brush

Laundry truck

Laundry truck

Canvass truck

Laundry tub

All-purpose truck

Tub

Wirehanger

Starchcooker

Netpins

Netbag

Earthenware receptacle

Container

Pail

Stacking table

Spotting kit

Vacuum

Laundry slip

Operation of washer

Load

Loading operation

Underloading

Overloading 
Charge réglementaire

Capacité maximale

Cycle de lavage

Déchargement

Essangeage

Programme de blanchissage

Programme accéléré

Acidification

Azurage

Bouillage

Empesage

Javelage

Rinçage

Rinçage à froid

Rinçage à chaud

Petit rinçage

Savonnage

Mousse abondante

Niveau de l'eau

Prérinçage

\section{Produits de lavage}

Acide

Acide acétique

Adoucisseur

Agent de blanchiment

Alcali

Amidon

Antirouille

Brillant

Blanchissant

Bleu

Bleu en cubes

Bleu en poudre

Bleu liquide

Bleu neutre

Bleu acide

Composé à base d'ammonium quaternaire

Détergent

Détersif

Détergent synthétique

Détacheur

Eau de Javel

Empois

Empois de blé

Empois de riz

Fluorure de silice

Fluorure acide de sodium

Hypochlorure de calcium

Hypochlorure de sodium

Métasilicate de sodium

Peroxyde d'hydrogène

Poudre à blanchir

Produit d'azurage
Correct loading

Maximum capacity

Wash cycle

Unloading operation

Soaking operation

Washfioor formula

Short formula

Souring operation

Bluing operation

Boil

Starching operation

Bleaching operation

Rinse

Cold rinse

Hot rinse

Flush

Suds operation

Heavy suds

Water level

Break operation

Washfloor supplies

Acid

Acetic acid

Water softener

Bleaching agent

Alcali

Raw starch

Rust remover

Brightener

Whitener

Laundry blue

Cake blue

Powdered blue

Liquid blue

Neutral blue

Acetic blue

Quaternary ammonium compound

Detergent

Detergent

Synthetic detergent

Spotting agent

Bleach

Starch

Wheat starch

Rice starch

Silico fluoride

Sodium acid fluoride

Calcium hypochloride

Sodium hypochloride

Sodium metasilicate

Hydrogen peroxide

Powdered bleach

Bluing agent 
Produit bactériostatique

Rigidifiant primaire

Savon

Savon à l'eau froide

Savon de faible teneur

Savon de forte teneur

Savon alcalinisé

Savon en pain

Savon en poudre

Savon en copeaux

Savon en paillettes

Savon neutre

Soude caustique

Acides

Propriétés des produits de lavage

Acidification

Action détersive

Action rapide

Efficacité de nettoyage

Déloger la saleté

Défloculation

Emulsionnant

Persistance de la blancheur

Mélange

Mise en suspension de la saleté

Pouvoir de dispersion

Pouvoir de mouillage

Pouvoir de neutralisation

Pouvoir de pénétration

Pur

Pourcentage de savon véritable

Saponification

Eau

Adoucisseur d'eau

Dureté de l'eau

Grains au gallon

Partie par million

Composé insoluble

Eau distillée

Eau douce

Eau dure

Eau froide

Eau tiède

Eau tempérée

Eau chaude

Eau bouillante

Fuites d'eau

Grains de dureté

$\mathrm{pH}$

Chiffre de $\mathrm{pH}$

pH-mètre

Savon de chaux

Sels minéraux
Bacteriostatic agent

Primary size

Soap

Cold water soap

Low titre soap

High titre soap

Built soap

Bar soap

Powdered soap

Flake soap (big flakes)

Flake soap (small flakes)

Neutral soap

Sodium hydroxide $(\mathrm{NaOH})$

Sours

Properties of washfloor supplies

Souring action

Detergent action

Quick action

Cleaning action

Dig out dirt

Defloculation

Emulsifier

Whiteness retention

Blend

Suspension of dirt particles

Dispersing power

Wetting power

Neutralizing power

Penetrating power

Without additives

$\%$ of actual soap

Saponification

Water

Watersoftener

Water hardness

Grains per gallon

Part per million

Insoluble compound

Distilled water

Soft water

Hard water

Cold water $\left(75-100^{\circ} \mathrm{F}\right)$

Lukewarm water $\left(100-120^{\circ} \mathrm{F}\right)$

Warm water $\left(120-140^{\circ} \mathrm{F}\right)$

Hot water $\left(140-180^{\circ} \mathrm{F}\right)$

Boiling hot water $\left(180-212^{\circ} \mathrm{F}\right)$

Leaks

Grains of hardness

$\mathrm{pH}$

$\mathrm{pH}$ value

$\mathrm{pH}$ meter

Limesoap

Mineral salts 
Sels de calcium

Sels de magnésium

Solution aqueuse

Solution de réserve

Solution savonneuse

Titrage

Vérification

Indicateur d'acide acétique d'acide chlorhydrique d'iodure de potassium de peroxyde d'hydrogène

Phénophtaléine

Rouge de méthyle

Solution de savon titré

\section{Linge d'hôpital}

Catégorie de linge

Linge plat

Linge en forme

Linge blanc

Linge de couleur

Classement $d u$ linge

Degré de saleté

Linge propre

Linge légèrement sale

Linge moyennement sale

Linge très sale

Linge pollué

\section{Couleurs}

Couleur grand (bon) teint

Couleurs qui déteignent

Teintes foncées

Teintes pâles

Manipulation du linge

Comptage

Circuit

Distribution

Entretien du linge

Marquage

Raccommodage

Ramassage

Rayonnage

Stockage

Stock dormant

Triage

Difficultés techniques

Azurage inégal

Décoloration

Dépôts de savon graisseux
Calcium salts

Magnesium salts

Aqueous solution

Stock solution

Soap solution

Titration

Test

Indicator

acetic acid

hydrochloric acid

potassium iodide

hydrogen peroxide

Phenolphtalein

Methyl red

Standard soap solution

Hospital linen

Main categories

Flatwork

Wearing apparel

White work

Colored work

Linen classification

Amount of soil

Clean linen

Slightly soiled linen

Medium soiled linen

Heavily soiled linen

Contaminated linen

Colors

Colorfast

Fugitives

Dark colors

Light colors

\section{Handling of linen}

Counting

Circulation

Distribution

Linen care

Marking

Linen repair

Collecting

Storing on shelves (temporarily)

Storage

Linen reserve

Sorting

Technical problems

Uneven bluing

Colorfading

Soap specs 
Dommage chimique

Dommage mécanique

Feutrage

Jaunissage

Odeur de linge propre

Perte de résistance à la tension

Rancir

Rétrécissement

Saleté incrustée

Taches

Taches de sang

Taches fixées

Tache de savon

Tache de graisse
Chemical damage

Mechanical damage

Felting

Yel'owing

Fresh smell

Loss of tensible strength

Turn rancid

Shrinkage

Ground-in dirt

Spots

Blood stains

Set stains

Soap spot

Grease spot

ERICH Oehnel

1. Jacques Surlemont, la Lettre commerciale, Bruxelles, Baude, 1945, p. 131.

2. Paul Robert, Dictionnaire alphabétique et analogique de la langue française, Paris, Société du Nouveau Littré, 1964, vol. VI, p. 51. 